\title{
EDITORIAL
}

\section{Therapy-related acute myeloid leukaemia with mutated NPM1: treatment induced or de novo in origin?}

Leukemia (2008) 22, 891-892; doi:10.1038/leu.2008.44

Acute myeloid leukaemia (AML) carrying NPM1 mutations that cause aberrant cytoplasmic expression of nucleophosmin, ${ }^{1,2}$ accounts for about one-third of de novo adult AML (50-60\% of AML with normal karyotype), and shows distinctive biological and clinical features. ${ }^{3}$ Until now, little information was available on the incidence of NPM1 mutations in therapyrelated AML (t-AML) or on the molecular and clinical characteristics of t-AML with mutated NPM1. ${ }^{1,4-6}$ Thus, the first comprehensive study of t-MDS (t-myelodysplasia)/t-AML with mutated NPM1 reported by Andersen et al. ${ }^{7}$ in this issue of the Journal is timely and remarkable.

Andersen et al. ${ }^{7}$ observed that NPM1 mutations occurred at lower frequency in t-AML than in de novo AML and were not related to any specific type of therapy. Notably, about $70 \%$ of the NPM1-positive t-MDS/t-AML had normal karyotype (an unusual finding in these cases, which generally show an abnormal karyotype) and none of them carried $7 q-1-7$, the most common abnormality in t-AML. ${ }^{8}$ Finally, about half of t-MDS/t-AML with mutated NPM1 expressed the FLT3 internal tandem duplication.

These findings are of great relevance since they indicate that t-AML with mutated NPM1 markedly differs cytogenetically and molecularly from other t-AML subtypes. Interestingly, normal karyotype and a high incidence of FLT3 internal tandem duplication are both distinctive features of NPM1-mutated AML with de novo origin. ${ }^{1}$ The finding by Andersen et al. ${ }^{7}$ that two t-AML with mutated NPM1 carried a ring chromosome 10 and a trisomy 8 , respectively, is in keeping with the observation that about $14 \%$ of de novo AML with mutated NPM1 harbour chromosomal aberrations, which are thought to be secondary. ${ }^{1}$

De novo AML with mutated NPM1 shows a distinct immunophenotype ${ }^{1}$ and gene expression profile, ${ }^{9-11}$ which is characterized by CD34 downregulation (at RNA and protein levels) and upregulation of most HOX genes. Unfortunately, Andersen et al. ${ }^{7}$ were not able to provide any information on the HOX gene signature and CD34 protein expression in their cases. However, the few NPM1-mutated t-AML with normal karyotype so far investigated were usually negative for the CD34 molecule, ${ }^{4}$ suggesting they may have the same immunophenotype (and possibly the same molecular signature) as NPM1-mutated AML arising de novo. ${ }^{1}$ Future efforts are warranted to clarify this issue.

Thus, although some information is still missing, the evidence strongly suggests that de novo and therapy-related NPM1mutated AML share common biological features. This finding is hardly surprising since t-AML with other recurrent genetic abnormalities, such as $t(15 ; 17)$ inv(16) or $t(8 ; 21)$, usually show the same biological and clinical findings as de novo AML with the corresponding karyotypes. ${ }^{12}$ Rather, it further supports the view that NPM1 mutation is a founder genetic alteration, which defines a distinct leukaemia entity. ${ }^{3}$
The results from Andersen et al. ${ }^{7}$ also raise the intriguing question of whether their t-AML cases with mutated NPM1 are truly secondary leukaemias induced by previous treatment or whether they represent de novo NPM1-mutated AML arising in patients with a history of therapy. If NPM1-mutated t-AML is related to previous treatment, the close association with normal karyotype clearly indicates that the leukaemogenic mechanisms must differ from those that operate in t-AML carrying an abnormal karyotype. In $\mathrm{t}-\mathrm{AML}$ with $\mathrm{t}(8 ; 21)$ or $\mathrm{t}(15 ; 17)$, DNA double breaks caused by drugs, especially topoisomerase II inhibitors, are thought to favour the development of balanced translocations. ${ }^{13,14}$ However, the role of NPM1 mutants in the development of de novo AML still remains to be elucidated, ${ }^{3}$ and, therefore, no causal relationship with previous treatment can be established at this time.

Conversely, like t-AML carrying internal-tandem duplication of the MLL gene, ${ }^{15}$ it is conceivable that a significant fraction of the t-AML reported by Andersen et al. ${ }^{7}$ are de novo leukaemias occurring incidentally in patients with a history of therapy. This is supported by the finding that these cases often received local radiotherapy or methotrexate plus prednisone, which are treatments whose leukaemogenic potential remains controversial. Moreover, two patients developed t-AML after unusually short or long latent periods. Another argument against these cases being secondary to therapy is that AML with mutated NPM1 has been constantly associated with a de novo origin. Indeed, NPM1 gene mutations are not usually observed in AML secondary to myelodysplasia or chronic myeloproliferative disorders, ${ }^{1,3}$ and in-depth analysis of the only reported case of NPM1-mutated AML in this setting revealed a possible de novo origin. $^{16}$

Therapy-related AML appears to be molecularly and clinically heterogeneous and its prognosis varies with the underlying genetic lesion. Cases harbouring $7 q-/-7$ genetic alterations are usually associated with poor prognosis, while t-AML carrying the $t(15 ; 17), t(8 ; 21)$ or inv(16) translocations generally have a good outcome. Thus, whatever the nature of NPM1-mutated t-AML (therapy-related or arising de novo), future clinical studies should address the issue of whether t-AML with the NPM1mutated/FLT3 internal tandem duplication-negative genotype shows the same favourable prognosis as the corresponding de novo cases.

\section{Acknowledgements}

This work was supported by the Associazione Italiana per la ricerca sul Cancro (AIRC). B Falini applied for a patent on the clinical use of NPM mutants.

B Falini

Section of Haematology and Immunology, Institute of Haematology, University of Perugia, Perugia, Italy E-mail: faliniem @unipg.it 


\section{References}

1 Falini B, Mecucci C, Tiacci E, Alcalay M, Rosati R, Pasqualucci L et al. Cytoplasmic nucleophosmin in acute myelogenous leukemia with a normal karyotype. N Engl J Med 2005; 352: 254-266.

2 Falini B, Bolli N, Shan J, Martelli MP, Liso A, Pucciarini A et al. Both carboxy-terminus NES motif and mutated tryptophan(s) are crucial for aberrant nuclear export of nucleophosmin leukemic mutants in NPMC+ AML. Blood 2006; 107: 4514-4523.

3 Falini B, Nicoletti I, Martelli MF, Mecucci C. Acute myeloid leukemia carrying cytoplasmic/mutated nucleophosmin (NPMc+ AML): biologic and clinical features. Blood 2007; 109: 874-885.

4 Schnittger S, Schoch C, Kern W, Mecucci C, Tschulik C, Martelli MF et al. Nucleophosmin gene mutations are predictors of favorable prognosis in acute myelogenous leukemia with a normal karyotype. Blood 2005; 106: 3733-3739.

5 Dohner K, Schlenk RF, Habdank M, Scholl C, Rucker FG, Corbacioglu A et al. Mutant nucleophosmin (NPM1) predicts favorable prognosis in younger adults with acute myeloid leukemia and normal cytogenetics: interaction with other gene mutations. Blood 2005; 106: 3740-3746.

6 Thiede C, Koch S, Creutzig E, Steudel C, Illmer T, Schaich M et al. Prevalence and prognostic impact of NPM1 mutations in 1485 adult patients with acute myeloid leukemia (AML). Blood 2006; 107: 4011-4020.

7 Andersen MT, Andersen MK, Christiansen DH, Pedersen-Bjergaard J. NPM1 mutations in therapy-related acute myeloid leukemia with uncharacteristic features. Leukemia 2008, e-pub ahead of print 14 February.

8 Pedersen-Bjergaard J, Andersen MT, Andersen MK. Genetic pathways in the pathogenesis of therapy-related myelodysplasia and acute myeloid leukemia. Hematology Am Soc Hematol Educ Program 2007; 2007: 392-397.

9 Alcalay $M$, Tiacci E, Bergomas R, Bigerna B, Venturini E, Minardi SP et al. Acute myeloid leukemia bearing cytoplasmic nucleophosmin (NPMc+ AML) shows a distinct gene expression profile characterized by up-regulation of genes involved in stem-cell maintenance. Blood 2005; 106: 899-902.

10 Verhaak RG, Goudswaard CS, van Putten W, Bijl MA, Sanders MA, Hugens $W$ et al. Mutations in nucleophosmin (NPM1) in acute myeloid leukemia (AML): association with other gene abnormalities and previously established gene expression signatures and their favorable prognostic significance. Blood 2005; 106: 3747-3754.

11 Mullighan CG, Kennedy A, Zhou X, Radtke I, Phillips LA, Shurtleff SA et al. Pediatric acute myeloid leukemia with NPM1 mutations is characterized by a gene expression profile with dysregulated HOX gene expression distinct from MLL-rearranged leukemias. Leukemia 2007; 21: 2000-2009.

12 Quesnel B, Kantarjian H, Bjergaard JP, Brault P, Estey E, Lai JL et al. Therapy-related acute myeloid leukemia with $\mathrm{t}(8 ; 21), \operatorname{inv}(16)$, and $\mathrm{t}(8 ; 16)$ : a report on 25 cases and review of the literature. J Clin Oncol 1993; 11: 2370-2379.

13 Zhang Y, Strissel P, Strick R, Chen J, Nucifora G, Le Beau MM et al. Genomic DNA breakpoints in AML1/RUNX1 and ETO cluster with topoisomerase II DNA cleavage and DNase I hypersensitive sites in t(8;21) leukemia. Proc Natl Acad Sci USA 2002; 99: 3070-3075.

14 Mistry AR, Felix CA, Whitmarsh RJ, Mason A, Reiter A, Cassinat B et al. DNA topoisomerase II in therapy-related acute promyelocytic leukemia. N Engl J Med 2005; 352: 1529-1538.

15 Christiansen DH, Pedersen-Bjergaard J. Internal tandem duplications of the FLT3 and MLL genes are mainly observed in atypical cases of therapy-related acute myeloid leukemia with a normal karyotype and are unrelated to type of previous therapy. Leukemia 2001; 15: 1848-1851.

16 Pasqualucci L, Li S, Meloni G, Schnittger S, Gattenlohner S, Liso A et al. NPM1-mutated acute myeloid leukaemia occurring in JAK2-V617F+ primary myelofibrosis: de-novo origin? Leukemia 2008, e-pub ahead of print 17 January. 